\title{
HAUSDORFF MEASURES AND THE MORSE-SARD THEOREM
}

\author{
Carlos Gustavo T. De A. Moreira
}

Abstract

Let $F: U \subset \mathbb{R}^{n} \rightarrow \mathbb{R}^{m}$ be a differentiable function and $p<m$ an integer. If $k \geq 1$ is an integer, $\alpha \in[0,1]$ and $F \in C^{k+(\alpha)}$, if we set $C_{p}(F)=\{x \in U \mid \operatorname{rank}(D f(x)) \leq p\}$ then the Hausdorff measure of dimension $\left(p+\frac{n-p}{k+\alpha}\right)$ of $F\left(C_{p}(F)\right)$ is zero.

\section{Introduction}

The Morse-Sard theorem is a fundamental theorem in analysis that is in the basis of transversality theory and differential topology. The classical Morse-Sard theorem states that the image of the set of critical points of a function $F: \mathbb{R}^{n} \rightarrow \mathbb{R}^{m}$ of class $C^{n-m+1}$ has zero Lebesgue measure in $\mathbb{R}^{m}$. It was proved by Morse $([\mathbf{M}])$ in the case $m=1$ and by Sard $([\mathbf{S} \mathbf{1}])$ in the general case.

Due to its theoretical importance, the Morse-Sard theorem was generalized in many directions. Many of these generalizations are related with Hausdorff measures and Hausdorff dimensions.

Given a metric space $X$ and a positive real number $\alpha$, we define the Hausdorff measure of dimension $\alpha$ associated to a covering $\mathcal{U}=\left(U_{\lambda}\right)_{\lambda \in L}$ of $X$ by bounded sets $U_{\lambda}$ by $m_{\alpha}(\mathcal{U})=\sum_{\lambda \in L}\left(\operatorname{diam} U_{\lambda}\right)^{\alpha}$, where $\operatorname{diam} U_{\lambda}$ denotes the diameter of $U_{\lambda}$, and, if we define the norm of a covering $\mathcal{U}$ by $\|\mathcal{U}\|=\sup _{U \in \mathcal{U}}(\operatorname{diam} U)$, then the Hausdorff measure of dimension $\alpha$ of $X$ is $m_{\alpha}(X)=\liminf _{\mathcal{U} \text { covering of } X} m_{\alpha}(\mathcal{U})$.

It is not difficult to see that there is a unique $d \in[0,+\infty]$ such that if $\alpha>d$ then $m_{\alpha}(X)=0$ and if $\alpha<d$ then $m_{\alpha}(X)=+\infty$. This number $d$ is called the Hausdorff dimension of $X$. It is easy to see that if $X \subset \mathbb{R}^{n}$ then its Hausdorff dimension $d=: H D(X)$ belongs to $[0, n]$. 
Sard himself proved that if $C_{p}(F)=\left\{x \in \mathbb{R}^{n} \mid \operatorname{rank}(D F(x)) \leq p\right\}$ then for any $\varepsilon>0$ there is $k \in \mathbb{N}$ such that if $F$ is $C^{k}$ then $F\left(C_{p}(F)\right)$ has zero Hausdorff measure of dimension $p+\varepsilon$ ([S2]). This result was made more precise by Federer $([\mathbf{F}])$, who proved that if $k \in \mathbb{N}$ then the Hausdorff measure of dimension $p+\frac{n-p}{k}$ of $F\left(C_{p}(F)\right)$ is zero. We should also mention the works of Church $([\mathbf{C h} \mathbf{1}],[\mathbf{C h} \mathbf{2}])$, which gave more results about the structure of the set of critical values of differentiable maps. Later, Yomdin $([\mathbf{Y}])$ proved that the Hausdorff dimension of $F\left(C_{p}(F)\right)$ is at most $p+\frac{n-p}{k+\alpha}$, provided that $F \in C^{k+\alpha}$, where $k \in \mathbb{N}$ and $0 \leq \alpha<1$. More recently, Bates ([B2]) proved that if $F \in C^{k+\alpha}$ with $k \in \mathbb{N}, 0<\alpha \leq 1$ and $p+\frac{n-p}{k+\alpha}=m$ then $F\left(C_{p}(F)\right)$ has zero Lebesgue measure in $\mathbb{R}^{m}$ (this in particular improves the hypothesis of the classical Morse-Sard theorem from $F \in C^{n-m+1}$ to $F \in C^{n-m+\text { Lips. }}$ i.e., $F \in C^{n-m}$ and $D^{n-m} F$ Lipschitz).

The aim of this work is to generalize the mentioned results by proving a general version of the Morse-Sard Theorem involving Hausdorff measures. Let $k \geq 1$ be an integer and $\alpha \in[0,1]$. We say that a function $F: U \subset \mathbb{R}^{n} \rightarrow \mathbb{R}^{m}$ is of class $C^{k+(\alpha)}$ at a subset $A$ of $U$ if $F$ is $C^{k}$ in $U$ and for each $x \in A$ there are $\varepsilon_{x}>0, K_{x}>0$ such that $|y-x|<\varepsilon_{x} \Rightarrow\left|D^{k} F(y)-D^{k} F(x)\right| \leq K_{x}|y-x|^{\alpha}$ (this is less restrictive than supposing $F \in C^{k+\alpha}$ ). Our main result is the following

Theorem. Let $F: U \subset \mathbb{R}^{n} \stackrel{C^{k}}{\longrightarrow} \mathbb{R}^{m}$ and let $p<m$ be an integer. If $C_{p}(F):=\{x \in U \mid \operatorname{rank}(D F(x)) \leq p\}$ and if $F$ is of class $C^{k+(\alpha)}$ at $C_{p}(F)$ then the Hausdorff $\left(p+\frac{n-p}{k+\alpha}\right)$-measure of $F\left(C_{p}(F)\right)$ is zero.

In particular, if $k+\alpha=\frac{n-p}{m-p}$, we recover the result of [B2], with a weaker hypothesis. We remark that if $p+\frac{n-p}{k+\alpha}<m$, the Hausdorff ( $p+$ $\frac{n-p}{k+\alpha}$ )-measure is not the Lebesgue measure or a product measure in $\mathbb{R}^{m}$, and so we can not use Fubini's Theorem. This difficulty is solved in the present paper by replacing the use of Fubini's theorem by a careful decomposition of the critical set, combined with a parametrized strong version of the main lemma of Morse's paper ([M, Theorem 2.1]).

We shall also give examples that show that our result is quite sharp, by giving counterexamples to slight changes of the hypothesis or of the conclusion.

\section{Functions whose zeros include a given set}

We shall prove here a version of Theorem 3.6 of [M] and Lemma 3.4.2 of $[\mathbf{F}]$, which will be fundamental for the later results. 
Theorem 2.1. Let $k \geq 1, \alpha \in[0,1], n>p$ and $A \subset U \subset \mathbb{R}^{n}$, where $U$ is an open set. Then there are sets $A_{1}, A_{2} \ldots \subset A$ such that $A=$ $\bigcup_{i=1}^{\infty} A_{i}$, where for each $i=1,2, \ldots$ there is a function $\psi_{i}: B_{i} \times V_{i} \stackrel{C^{1}}{\longrightarrow} U$ where $B_{i}$ is a ball in some $\mathbb{R}^{r_{i}}, r_{i} \geq 0$ and $V_{i}$ is a ball in $\mathbb{R}^{p}$ such that $\psi_{i}(x, y)=\left(\widetilde{\psi}_{i}(x, y), y\right)$, and $\left|\psi_{i}\left(x_{1}, y_{1}\right)-\psi_{i}\left(x_{2}, y_{2}\right)\right| \geq\left|\left(x_{1}, y_{1}\right)-\left(x_{2}, y_{2}\right)\right|$, $\forall\left(x_{1}, y_{1}\right),\left(x_{2}, y_{2}\right) \in B_{i} \times V_{i}$ and $A_{i} \subset \psi_{i}\left(B_{i} \times V_{i}\right)$, with the following property: We can write $A_{i}=A_{i}^{\prime} \cup A_{i}^{\prime \prime}$ so that $\psi_{i}^{-1}\left(A_{i}^{\prime \prime}\right)$ has measure zero in $B_{i} \times V_{i}$, and if $f: U \rightarrow \mathbb{R}$ vanishes in $A$ and $f$ is $C^{k+(\alpha)}$ at $A$ we have:

- $\limsup _{\left(x, y_{0}\right) \rightarrow\left(x_{0}, y_{0}\right)} \frac{f\left(\psi_{i}\left(x, y_{0}\right)\right)}{\left|x-x_{0}\right|^{k+\alpha}}<+\infty, \forall\left(x_{0}, y_{0}\right) \in B_{i} \times V_{i}$ such that $\psi_{i}\left(x_{0}, y_{0}\right) \in A_{i}$,

- $\lim _{\left(x, y_{0}\right) \rightarrow\left(x_{0}, y_{0}\right)} \frac{f\left(\psi_{i}\left(x, y_{0}\right)\right)}{\left|x-x_{0}\right|^{k+\alpha}}=0, \forall\left(x_{0}, y_{0}\right) \in B_{i} \times V_{i}$ such that $\left.\psi_{i}\left(x_{0}, y_{0}\right)\right) \in A_{i}^{\prime}$.

Proof: Let us consider first the case $k=1$ and $d f(x) \cdot v=0 \forall x \in A$, $v \in \mathbb{R}^{n-p} \times\{0\}$. In this case we take $A=\left(A^{\prime} \cap A\right) \cup A^{\prime \prime}$ where $A^{\prime}$ is the set of density points of $\bar{A}$ in the direction of $\mathbb{R}^{n-p} \times\{0\} \quad((x, y) \in$ $A^{\prime} \Rightarrow \lim _{\varepsilon \rightarrow 0} \frac{m\left(\left(B_{\varepsilon}(x) \times\{y\}\right) \cap \bar{A}\right)}{m\left(B_{\varepsilon}(x)\right)}=1$, where $m$ is the $(n-p)$-dimensional measure). The measure of $A^{\prime \prime}=A-A^{\prime}$ is zero, since it is zero in each plane $\mathbb{R}^{n-p} \times\{y\}$.

For $\left(x_{0}, y_{0}\right) \in A$ take $B\left(\left(x_{0}, y_{0}\right), \varepsilon\left(x_{0}, y_{0}\right)\right)$ a ball contained in $U$ and $\psi=\left.\operatorname{Id}\right|_{B\left(\left(x_{0}, y_{0}\right), \varepsilon\left(x_{0}, y_{0}\right)\right)}$. We have $\limsup _{\left(x, y_{0}\right) \rightarrow\left(x_{0}, y_{0}\right)} \frac{f\left(x, y_{0}\right)}{\left|x-x_{0}\right|^{1+\alpha}}<+\infty$, since $f\left(x, y_{0}\right)=f\left(x, y_{0}\right)-f\left(x_{0}, y_{0}\right)=d f\left(t x_{0}+(1-t) x\right)\left(x-x_{0}\right), t \in$ $(0,1) \Rightarrow\left|f\left(x, y_{0}\right)\right| \leq K_{x_{0}}\left|x-x_{0}\right|^{1+\alpha}$. For $\left(x_{0}, y_{0}\right) \in A^{\prime}$,

$$
\lim _{\delta \rightarrow 0} \frac{1}{\operatorname{vol}\left(S^{n-p-1}\right)} \int_{S^{n-p-1}}\left(\frac{1}{\delta} \int_{0}^{\delta} \chi_{\bar{A}}\left(x_{0}+t v, y_{0}\right) d t\right) d v=1,
$$

so $\forall \varepsilon>0 \exists \delta_{0}>0$ s.t. $\left|x-x_{0}\right|<\delta_{0} \Rightarrow \exists v \in S^{n-p-1}$ with

$$
\left|v-\frac{x-x_{0}}{\left|x-x_{0}\right|}\right|<\varepsilon
$$

and

$$
\left|\frac{1}{\left|x-x_{0}\right|} \int_{0}^{\left|x-x_{0}\right|} \chi_{\bar{A}}\left(x_{0}+t v, y_{0}\right) d t-1\right|<\varepsilon,
$$


so, if $\tilde{x}=x_{0}+\left|x-x_{0}\right| v$,

$$
\left|f\left(x, y_{0}\right)-f\left(x_{0}, y_{0}\right)\right| \leq\left|f\left(x, y_{0}\right)-f\left(\tilde{x}, y_{0}\right)\right|+\left|f\left(\tilde{x}, y_{0}\right)-f\left(x_{0}, y_{0}\right)\right|,
$$

but

$$
\begin{aligned}
& f\left(x, y_{0}\right)-f\left(\tilde{x}, y_{0}\right)=d f\left(\theta t+(1-\theta) \tilde{x}, y_{0}\right) \cdot(x-\tilde{x}), \quad \theta \in(0,1) \\
\Rightarrow & \left|f\left(x, y_{0}\right)-f\left(\tilde{x}, y_{0}\right)\right| \leq K_{x_{0}}\left|x-x_{0}\right|^{\alpha} \cdot \varepsilon\left|x-x_{0}\right|=\varepsilon K_{x_{0}}\left|x-x_{0}\right|^{1+\alpha}
\end{aligned}
$$

and

$$
\begin{aligned}
f\left(\tilde{x}, y_{0}\right) & -f\left(x_{0}, y_{0}\right) \\
& =\int_{0}^{\left|\tilde{x}-x_{0}\right|} d f\left(x_{0}+t v, y_{0}\right) \cdot v d t \\
& \leq K_{x_{0}}\left|x-x_{0}\right|^{\alpha} \cdot m\left\{t \in\left[0,\left|\tilde{x}-x_{0}\right|\right] \mid \frac{\partial f}{\partial x}\left(x_{0}+t v, y_{0}\right) \neq 0\right\} \\
& \leq K_{x_{0}}\left|x-x_{0}\right|^{\alpha} \cdot \varepsilon\left|x-x_{0}\right|=\varepsilon K_{x_{0}}\left|x-x_{0}\right|^{1+\alpha} .
\end{aligned}
$$

So

$$
\begin{aligned}
\left|f\left(x, y_{0}\right)\right|=\left|f\left(x, y_{0}\right)-f\left(x_{0}, y_{0}\right)\right| \leq & 2 \varepsilon K_{x_{0}}\left|x-x_{0}\right|^{1+\alpha} \\
& \Rightarrow \lim _{\left(x, y_{0}\right) \rightarrow\left(x_{0}, y_{0}\right)} \frac{f\left(x, y_{0}\right)}{\left|x-x_{0}\right|^{1+\alpha}}=0 .
\end{aligned}
$$

We can take a countable subcovering of $A$ by the $B\left(\left(x_{0}, y_{0}\right), \varepsilon\left(x_{0}, y_{0}\right)\right)$ to finish the proof in this case.

Consider now the case $k \geq 1, n$ arbitrary. We have $A=A^{*} \cup A^{* *}$ where $A^{*}=\left\{x \in A\left|\exists g: U \stackrel{C^{k}}{\longrightarrow} \mathbb{R}, g\right|_{A} \equiv 0, \exists v \in \mathbb{R}^{n-p} \times\{0\}\right.$, $d g(x) \cdot v \neq 0\} . \quad A^{* *}=A \backslash A^{*}$. If $\left(x_{0}, y_{0}\right) \in A^{*}$ there is $g$ as above, so there is $\varepsilon>0$ such that $g^{-1}(0) \cap B_{\varepsilon}\left(x_{0}, y_{0}\right)$ is contained in the image of $\psi: B \times V \stackrel{C^{k}}{\longrightarrow} U$ where $B$ is a ball in $\mathbb{R}^{n-p-1}$, as in the statement, and $A \subset g^{-1}(0)$. Taking a countable subcovering of $A^{*}$ by these balls we reduce the proof in this case to a case with smaller $n$. If $k=1$, the result was yet proved for $A^{* *}$. If $k>1$, and assuming by induction the result for $k-1$, we have

$$
A^{* *}=\bigcup_{i=1}^{\infty} A_{i}^{* *}, A_{i}^{* *}=\left(A_{i}^{* *}\right)^{\prime} \cup\left(A_{i}^{* *}\right)^{\prime \prime}, A_{i}^{* *} \subset \psi_{i}\left(B_{i} \times V_{i}\right), \quad \psi_{i} \in C^{1},
$$




$$
\begin{aligned}
\psi_{i}\left(x_{0}, y_{0}\right) \in A_{i}^{* *} & \Rightarrow \limsup _{x \rightarrow x_{0}} \frac{\left.|| d f\left(\psi_{i}\left(x, y_{0}\right)\right)\right|_{\mathbb{R}^{n-p} \times\{0\}}||}{\left|x-x_{0}\right|^{k-1+\alpha}}<+\infty \\
& \Rightarrow \limsup _{x \rightarrow x_{0}} \frac{\left|f\left(\psi_{i}\left(x, y_{0}\right)\right)\right|}{\left|x-x_{0}\right|^{k+\alpha}}<+\infty
\end{aligned}
$$

and

$$
\begin{aligned}
\psi_{i}\left(x_{0}, y_{0}\right) \in\left(A_{i}^{* *}\right) & \Rightarrow \lim _{x \rightarrow x_{0}} \frac{\left\|\left.d f\left(\psi_{i}\left(x, y_{0}\right)\right)\right|_{\mathbb{R}^{n-p} \times\{0\}}\right\|}{\left|x-x_{0}\right|^{k-1+\alpha}}=0 \\
& \Rightarrow \lim _{x \rightarrow x_{0}} \frac{f\left(\psi_{i}\left(x, y_{0}\right)\right)}{\left|x-x_{0}\right|^{k+\alpha}}=0
\end{aligned}
$$

both by the mean value theorem, and the proof is finished by induction.

Corollary 2.2. Let $k \geq 1, \alpha \in[0,1], n>p$ and $A \subset U \subset \mathbb{R}^{n}$, where $U$ is an open set. Then there are sets $A_{1}, A_{2} \ldots \subset A$ such that $A=$ $\bigcup_{i=1}^{\infty} A_{i}$, where for each $i=1,2, \ldots$ there is a function $\psi_{i}: B_{i} \times V_{i} \stackrel{C^{1}}{\longrightarrow} U$ where $B_{i}$ is a ball in some $\mathbb{R}^{r_{i}}, r_{i} \geq 0$ and $V_{i}$ is a ball in $\mathbb{R}^{p}$ such that $\psi_{i}(x, y)=\left(\widetilde{\psi}_{i}(x, y), y\right)$, and $\left|\psi_{i}\left(x_{1}, y_{1}\right)-\psi_{i}\left(x_{2}, y_{2}\right)\right| \geq\left|\left(x_{1}, y_{1}\right)-\left(x_{2}, y_{2}\right)\right|$, $\forall\left(x_{1}, y_{1}\right),\left(x_{2}, y_{2}\right) \in B_{i} \times V_{i}$ and $A_{i} \subset \psi_{i}\left(B_{i} \times V_{i}\right)$, with the following property: We can write $A_{i}=A_{i}^{\prime} \cup A_{i}^{\prime \prime}$ so that $\psi_{i}^{-1}\left(A_{i}^{\prime \prime}\right)$ has measure zero in $B_{i} \times V_{i}$, and if $f: U \rightarrow \mathbb{R}$ is $C^{k+(\alpha)}$ at $A$ and $D_{x} f \equiv 0$ in $A$ we have:

- $\limsup _{\left(x, y_{0}\right) \rightarrow\left(x_{0}, y_{0}\right)} \frac{\left|f\left(\psi_{i}\left(x, y_{0}\right)\right)-f\left(\psi_{i}\left(x_{0}, y_{0}\right)\right)\right|}{\left|x-x_{0}\right|^{k+\alpha}}<+\infty, \forall\left(x_{0}, y_{0}\right) \in B_{i} \times$ $V_{i}$ such that $\psi_{i}\left(x_{0}, y_{0}\right) \in A_{i}$,

- $\lim _{\left(x, y_{0}\right) \rightarrow\left(x_{0}, y_{0}\right)} \frac{\left|f\left(\psi_{i}\left(x, y_{0}\right)\right)-f\left(\psi_{i}\left(x_{0}, y_{0}\right)\right)\right|}{\left|x-x_{0}\right|^{k+\alpha}}=0, \forall\left(x_{0}, y_{0}\right) \in B_{i} \times V_{i}$ such that $\left.\psi_{i}\left(x_{0}, y_{0}\right)\right) \in A_{i}^{\prime}$.

Proof: If $k \geq 2$ this is an immediate consequence of Theorem 2.1 applied to $D_{x} f$ and of the mean value theorem. If $k=1$ this can be proved exactly as the case $k=1$ of the Theorem 2.1 .

Corollary 2.3. In the statements of Theorem 2.1 and Corollary 2.2, for any $x \in B_{i}$ s.t. $\psi_{i}(x) \in A_{i}$ there are $\varepsilon_{x}>0, K_{x}>0$ such that $|y-x|<\varepsilon_{x} \Rightarrow\left|f\left(\psi_{i}(y)\right)-f\left(\psi_{i}(x)\right)\right| \leq K_{x}|y-x|^{k+\alpha}$, and for any $\varepsilon>0$ there is a $\delta>0$ so that $\frac{\lambda\left(\psi_{i}^{-1}\left(A_{i}\right) \cap B_{r}(x)\right)}{\lambda\left(B_{r}(x)\right)}>1-\delta \Rightarrow\left|f\left(\psi_{i}(y)\right)-f\left(\psi_{i}(x)\right)\right| \leq$ $\varepsilon K_{x} r^{k+\alpha}$, if $r \leq \varepsilon_{x}$ and $|y-x| \leq r$ ( $\delta$ depends only on $\varepsilon$ and $n$, but not on $f$ or on $x$ ). 
Proof: This is only a more precise formulation of the results proved in the demonstration of the theorem.

Remark 2.1. For $k=0$ we have the same results, except the statement $\lim _{y \rightarrow x} \frac{f\left(\psi_{i}(y)\right)}{|y-x|^{k+\alpha}}=0$, for each $x \in B_{i}$ such that $\psi_{i}(x) \in A_{i}^{\prime}$.

\section{The main results}

Lemma 3.1. Let $A \subset \mathbb{R}^{m}$ with $\lambda(A)<\infty$ and let $\mathcal{U}$ be a family of balls $B_{r}(x), x \in A$ such that for each $x \in A$ there is an $\varepsilon_{x}>0$ such that $r \leq \varepsilon_{x} \Rightarrow B_{r}(x) \in \mathcal{U}$. Then for each $\varepsilon>0$ there are $x_{n} \in A, r_{n}>0$ with $B_{r_{n}}\left(x_{n}\right) \in \mathcal{U}$ and $A \subset \bigcup_{n=1}^{\infty} B_{r_{n}}\left(x_{n}\right)$ such that $\sum_{n=1}^{\infty} \lambda\left(B_{r_{n}}\left(x_{n}\right)\right)<$ $\lambda(A)+\varepsilon$.

Proof: This lemma is essentially the Vitali covering theorem from measure theory. Take $U \supset A$ an open set with $\lambda(U)<\lambda(A)+\frac{\varepsilon}{2}$. If we choosed $B_{\tilde{r}_{1}}\left(x_{1}\right), \ldots, B_{\tilde{r}_{n}}\left(x_{n}\right)$, define $s_{n}=\sup \{r>0 \mid \exists x \in A$ s.t. $r<$ $\frac{\varepsilon_{x}}{5}, B_{r}(x) \subset U$ and $\left.B_{r}(x) \cap\left(B_{\tilde{r}_{1}}\left(x_{1}\right) \cup \cdots \cup B_{\tilde{r}_{n}}\left(x_{n}\right)\right)=\emptyset\right\}$. Choose $B_{\tilde{r}_{n+1}}\left(x_{n+1}\right)$ such that $\tilde{r}_{n+1}>\frac{s_{n}}{2}, \tilde{r}_{n+1}<\frac{\varepsilon_{x_{n+1}}}{5}, B_{\tilde{r}_{n+1}}\left(x_{n+1}\right) \subset U$ and $B_{\tilde{r}_{n+1}}\left(x_{n+1}\right) \cap\left(B_{\tilde{r}_{1}}\left(x_{1}\right) \cup \cdots \cup B_{\tilde{r}_{n}}\left(x_{n}\right)\right)=\emptyset$. Since the $B_{\tilde{r}_{i}}\left(x_{i}\right)$ are disjoint and contained in $U$ we have $\sum_{i=1}^{\infty} \lambda\left(B_{\tilde{r}_{i}}\left(x_{i}\right)\right)<\lambda(A)+\frac{\varepsilon}{2}$, and so there is a $n_{0} \in \mathbb{N}$ such that $\sum_{i=n_{0}}^{\infty} \lambda\left(B_{5 \tilde{r}_{i}}\left(x_{i}\right)\right)<\frac{\varepsilon}{2}$. We take $B_{r_{i}}\left(x_{i}\right)=B_{\tilde{r}_{i}}\left(x_{i}\right)$, $i<n_{0}$ and $B_{r_{i}}\left(x_{i}\right)=B_{5 \tilde{r}_{i}}\left(x_{i}\right), i \geq n_{0}$.

Clearly we have $\sum_{i=1}^{\infty} \lambda\left(B_{r_{i}}\left(x_{i}\right)\right)<\lambda(A)+\varepsilon$. To prove that $A \subset$ $\bigcup_{n=1}^{\infty} \overline{B_{r_{n}}\left(x_{n}\right)}$, take $x \in A$ and $r=\min \left\{\tilde{r}_{n_{0}}, \varepsilon_{x} / 5, d\left(x, U^{c} \cup \bigcup_{i<n_{0}} B_{r_{i}}\left(x_{i}\right)\right)\right\}$. If $r>0$, take $n \geq n_{0}$ such that $s_{n}<r \leq s_{n-1}$ (we have $r \leq \tilde{r}_{n_{0}} \leq s_{n_{0}-1}$ ), and note that $s_{n}<r \Rightarrow B_{r}(x) \cap\left(B_{\tilde{r}_{1}}\left(x_{1}\right) \cup \cdots \cup B_{\tilde{r}_{n}}\left(x_{n}\right)\right) \neq \emptyset \Rightarrow \exists i \leq n$ such that $B_{r}(x) \cap B_{\tilde{r}_{i}}\left(x_{i}\right) \neq \emptyset$. We have $n \geq n_{0}$ since $r \leq d\left(x, B_{\tilde{r}_{i}}\left(x_{i}\right)\right)$, and $\tilde{r}_{i}>\frac{s_{n-1}}{2} \geq \frac{r}{2}$, since $i \leq n$. Therefore, we have $x \in B_{5 \tilde{r}_{i}}\left(x_{i}\right)$. If $r=0$ then $x \in \overline{B_{r_{i}}\left(x_{i}\right)}$ for some $i<n_{0}$. This proves that $A \subset \bigcup_{n=1}^{\infty} \overline{B_{r_{n}}\left(x_{n}\right)}$. Taking $\tilde{r}_{n}=\left(\frac{\lambda(A)+\varepsilon}{\sum_{i=1}^{\infty} \lambda\left(B_{r_{i}}\left(x_{i}\right)\right)}\right)^{1 / 2 m} \cdot r_{n}$, we have $A \subset \bigcup_{n=1}^{\infty} B_{\tilde{r}_{n}}\left(x_{n}\right)$, with $\sum_{n=1}^{\infty} \lambda\left(B_{\tilde{r}_{n}}\left(x_{n}\right)\right)=(\lambda(A)+\varepsilon)^{1 / 2}\left(\sum_{i=1}^{\infty} \lambda\left(B_{r_{i}}\left(x_{i}\right)\right)\right)^{1 / 2}<\lambda(A)+\varepsilon$.

Remark 3.1. In the Lemma 3.1 we can replace a family of balls $B_{r}(x)$ by a family of cubes $C_{r}(x)=\prod_{i=1}^{m}\left[x_{i}-r, x_{i}+r\right]$, where $x=\left(x_{1}, \ldots, x_{m}\right)$, using the same proof.

Lemma 3.2. Let $F: U \subset \mathbb{R}^{n} \rightarrow \mathbb{R}^{m}$ be a function, $A \subset U$ and $d>0$ such that for any $x \in A$ there are $\varepsilon_{x}>0, K_{x}>0$ such that $m_{d}\left(F\left(B_{\varepsilon}(x) \cap\right.\right.$ $A)) \leq K_{x} \cdot \lambda\left(B_{\varepsilon}(x)\right), \forall \varepsilon<\varepsilon_{x}$, where $m_{d}$ is the Hausdorff measure of dimension $d$, and there is $A^{\prime} \subset A$ such that $\lambda\left(A \backslash A^{\prime}\right)=0$ and $\lim _{\varepsilon \rightarrow 0} \frac{m_{d}\left(F\left(B_{\varepsilon}(x) \cap A\right)\right)}{\lambda\left(B_{\varepsilon}(x)\right)}=0, \forall x \in A^{\prime}$. Then $m_{d}(F(A))=0$. 
Remark 3.2. The same result is true if we replace $B_{\varepsilon}(x)$ by $C_{\varepsilon}(x)$.

Remark 3.3. We can replace the condition

$$
" m_{d}\left(F\left(B_{\varepsilon}(x) \cap A\right)\right) \leq K_{x} \lambda\left(B_{\varepsilon}(x)\right), \quad \forall \varepsilon<\varepsilon_{x} "
$$

by

" $F\left(B_{\varepsilon}(x) \cap A\right)$ can be covered by balls $B_{\delta_{i}}\left(y_{i}\right), \quad i \in \mathbb{N}$,

$$
\text { with } \sum_{i=1}^{\infty} \delta_{i}^{d} \leq K_{x} \lambda\left(B_{\varepsilon}(x)\right), \quad \forall \varepsilon<\varepsilon_{x} ",
$$

and the condition

$$
" \lim _{\varepsilon \rightarrow 0} \frac{m_{d}\left(F\left(B_{\varepsilon}(x) \cap A\right)\right.}{\lambda\left(B_{\varepsilon}(x)\right)}=0, \quad \forall x \in A^{\prime} "
$$

by

" $F\left(B_{\varepsilon}(x) \cap A\right)$ can be covered by balls $B_{\delta_{i}^{(\varepsilon)}}\left(y_{i}\right), \quad i \in \mathbb{N}$

$$
\text { with } \lim _{\varepsilon \rightarrow 0} \frac{\sum_{i=1}^{\infty}\left(\delta_{i}^{(\varepsilon)}\right)^{d}}{\lambda\left(B_{\varepsilon}(x)\right)}=0, \quad \forall x \in A^{\prime \prime} .
$$

The proof remains essentially the same, and Remark 3.2 is still valid.

Remark 3.4. If we replace the conditions of this lemma by " $F\left(B_{\varepsilon}(x) \cap A\right)$ can be covered by balls $B_{\delta_{i}}\left(y_{i}\right), i \in \mathbb{N}$, with $\sum_{i=1}^{\infty} \delta_{i}^{d} \leq k \lambda\left(B_{\varepsilon}(x)\right)$, $\forall \varepsilon<\varepsilon_{x}$ (note that here $k$ does not depend on $x$ ), and $\lambda(\bar{A})<\infty$ ", then we can conclude, using the same proof, that $m_{d}(F(A)) \leq k \lambda(A)$.

Proof: We may suppose that $A$ has finite Lebesgue measure, since $A$ is a countable union of sets with finite measure, and a countable union of sets with Hausdorff $d$-measure zero has Hausdorff $d$-measure zero. Moreover, since $A=\bigcup_{k=1}^{\infty} A_{k}$, where $A_{k}=\left\{x \in A \mid K_{x} \leq k\right\}$, we may suppose $K_{x} \leq K, \forall x \in A$. Let $C$ be the Lebesgue measure of $A$.

Let $\varepsilon>0$. For each $x \in A^{\prime}$ take $\delta_{x}>0$ such that $B_{\delta_{x}}(x) \subset U$ and $r \leq \delta_{x} \Rightarrow \frac{m_{d}\left(F\left(B_{r}(x) \cap A\right)\right)}{\lambda\left(B_{r}(x)\right)} \leq \frac{\varepsilon}{2(C+1)}$. By the Lemma 3.1 we can cover $A^{\prime}$ by $\bigcup_{n=1}^{\infty} B_{r_{n}}\left(x_{n}\right)$ with

$$
\sum_{n=1}^{\infty} \lambda\left(B_{r_{n}}\left(x_{n}\right)\right)<C+1
$$


and

$$
\begin{aligned}
r_{n} & \leq \delta_{x_{n}} \Rightarrow \sum_{n=1}^{\infty} m_{d}\left(F\left(B_{r_{n}}\left(x_{n}\right) \cap A\right)\right) \\
& \leq \frac{\varepsilon}{2(C+1)} \cdot(C+1)=\frac{\varepsilon}{2} \Rightarrow m_{d}\left(F\left(A^{\prime}\right)\right) \leq \frac{\varepsilon}{2} .
\end{aligned}
$$

By Lemma 3.1 we can cover $A \backslash A^{\prime}$ by $\bigcup_{n=1}^{\infty} B_{\tilde{r}_{n}}\left(\widetilde{x}_{n}\right)$ such that $B_{\tilde{r}_{n}}\left(\widetilde{x}_{n}\right) \subset$ $U$ and $\tilde{r}_{n}<\varepsilon_{\widetilde{x}_{n}}, \forall n \in \mathbb{N}$, with

$$
\begin{aligned}
\sum_{n=1}^{\infty} \lambda\left(B_{\tilde{r}_{n}}\left(\widetilde{x}_{n}\right)\right) & <\frac{\varepsilon}{2 K} \Rightarrow \sum_{n=1}^{\infty} m_{d}\left(F\left(B_{\tilde{r}_{n}}\left(\widetilde{x}_{n}\right)\right)\right) \\
& \leq \frac{\varepsilon}{2 K} \cdot K=\frac{\varepsilon}{2} \Rightarrow m_{d}\left(F\left(A \backslash A^{\prime}\right)\right) \\
& \leq \frac{\varepsilon}{2} \Rightarrow m_{d}(F(A)) \leq \frac{\varepsilon}{2}+\frac{\varepsilon}{2}=\varepsilon .
\end{aligned}
$$

Since $\varepsilon>0$ is arbitrary we have $m_{d}(F(A))=0$.

We first use Lemma 3.2 to prove the following strong version of Constantin's result $([\mathbf{C o}])$, that does not suppose continuity of the derivatives. Here we do not suppose differentiability in every point, but only in the set of critical points under consideration.

Theorem 3.3. Let $F: X \subset \mathbb{R}^{n} \rightarrow \mathbb{R}^{n}$ be a function, and let $A=\{x \in$ $X \mid D F(x)$ exists and is not surjective $\}$. Then $\lambda(F(A))=0$.

Proof: It is a simple consequence of Lemma 3.2, since if $x \in A$ then $\lim _{r \rightarrow 0} \frac{\lambda\left(F\left(B_{r}(x)\right)\right)}{\lambda\left(B_{r}(x)\right)}=0$. Indeed, $x \in A \Rightarrow F(x+h)=F(x)+D F(x) . h+$ $r(h)$, where $\lim _{h \rightarrow 0} \frac{r(h)}{|h|}=0$. Let $K=\|D F(x)\|$, and let $\varepsilon \in(0,1)$. Let $\delta>0$ such that $|h| \leq \delta \Rightarrow \frac{|r(h)|}{|h|}<\frac{\varepsilon}{2(K+1)^{n-1}}$. Then, if $|h| \leq \delta$, $F(x+h)-F(x)$ belongs to an $\frac{\varepsilon \cdot|h|}{2(K+1)^{n-1}}$ neighbourhood of a ball of radius $K|h|$ in a subspace of $\mathbb{R}^{n}$ of dimension $n-1$ (a fixed subspace of $\mathbb{R}^{n}$ of dimension $n-1$ which contains the image of $D F$ ), and thus belongs to the orthogonal product of a ball of radius $(K+1)|h|$ in this subspace by an interval of radius $\frac{\varepsilon|h|}{2(K+1)^{n-1}}$. Therefore, $\lambda\left(F\left(B_{r}(x)\right) \leq\right.$ $\frac{\varepsilon . r . r^{n-1}(K+1)^{n-1}}{(K+1)^{n-1}} v_{n-1}=\varepsilon r^{n} v_{n-1}$, where $v_{n-1}$ is the volume of the unitary ball in $\mathbb{R}^{n-1}$, and, since $\varepsilon>0$ is arbitrary, $\lim _{r \rightarrow 0} \frac{\lambda\left(F\left(B_{r}(x)\right)\right)}{\lambda\left(B_{r}(x)\right)}=0$.

Theorem 3.4. Let $F: U \subset \mathbb{R}^{n} \stackrel{C^{k}}{\longrightarrow} \mathbb{R}^{m}$ be a function of class $C^{k+(\alpha)}(\alpha \in$ $(0,1])$ at $C_{p}(F):=\{x \in U \mid \operatorname{rank}(D F(x)) \leq p\}$. Then the Hausdorff measure of dimension $d=p+\frac{n-p}{k+\alpha}$ of $F\left(C_{p}(F)\right)$ is zero, $\forall p<\min \{m, n\}$. 
Proof: Since $C_{p}(F)=\bigcup_{r=0}^{p}\{x \in U \mid \operatorname{rank}(D F(x))=r\}$, and $r+\frac{n-r}{k+\alpha} \leq$ $p+\frac{n-p}{k+\alpha}$ for $0 \leq r \leq p$, we may restrict our attention to $\widetilde{C}_{p}(F)=\{x \in$ $U \mid \operatorname{rank}(D F(x))=p\}$. If $x_{0} \in C_{p}(F)$, we have, after a change of coordinates of class $C^{k}, F(z, y)=(z, G(z, y))$, with $(z, y) \in \mathbb{R}^{p} \times \mathbb{R}^{n-p}$ and $G(z, y) \in \mathbb{R}^{m-p}$, in a neighbourhood $V$ of $x_{0}=\left(z_{0}, y_{0}\right)$. We shall restrict our attention to this neighbourhood. We have $x=(z, y) \in$ $C_{p}(F) \Leftrightarrow D_{y} G(z, y)=0$. We can apply the results of the Section 2 (Theorem 2.1, Corollary 2.3 and Remark 2.1) to the function $D_{y} G$, and obtain the decomposition $A=\bigcup_{i=1}^{\infty} A_{i}, A_{i} \subset \psi_{i}\left(V_{i} \times B_{i}\right)$, where $A=\left\{(z, y) \in V \mid D_{y} G(z, y)=0\right\}$. Let us fix such an $A_{i}$.

Since $\psi_{i}^{-1}\left(A_{i}\right)=\bigcup_{m \in \mathbb{N}}\left\{x \in \psi_{i}^{-1}\left(A_{i}\right) \mid \varepsilon_{x} \geq \frac{1}{m}, K_{x} \leq m\right\}$, we may suppose $\varepsilon_{x} \geq \frac{1}{M}, K_{x} \leq M, \forall x \in \psi_{i}^{-1}\left(A_{i}\right)$, for some fixed $M$ and also that $V$ has finite Lebesgue measure $\lambda(V)$.

With these assumptions, we shall prove that there is a constant $K_{0}$ such that for any $X \subset V, \nu>0$, we can cover $F\left(A_{i} \cap X\right)$ by balls $B_{\delta_{i}}\left(p_{i}\right)$ so that $\sum_{i=1}^{\infty} \delta_{i}^{d} \leq K_{0}(\lambda(X)+\nu)$. For this, given a point $x \in A_{i} \cap X$ and an $\varepsilon<\frac{1}{2 \sqrt{n} M}$, we can divide the cube $C_{\varepsilon}(x)=C_{\varepsilon}(z) \times C_{\varepsilon}(y)$ into $\left(\left[\varepsilon^{1-(k+\alpha)}\right]+1\right)^{p}$ boxes $C_{\delta}\left(\widetilde{z}_{i}\right) \times C_{\varepsilon}(y), \delta<\varepsilon^{k+\alpha}$. If there is some point $\left(z_{i}, y_{i}\right)$ in $\left(C_{\delta}\left(\widetilde{z}_{i}\right) \times C_{\varepsilon}(y)\right) \cap\left(A_{i} \cap X\right)$, then for any point $\left(z_{i}^{\prime}, y_{i}^{\prime}\right)$ in $\left(C_{\delta}\left(\widetilde{z}_{1}\right) \times C_{\varepsilon}(y)\right) \cap\left(A_{i} \cap X\right)$, we have $\left|F\left(z_{i}^{\prime}, y^{\prime}\right)-F\left(z_{i}, y_{i}\right)\right| \leq \mid F\left(z_{i}^{\prime}, y^{\prime}\right)-$ $F\left(z_{i}, y^{\prime}\right)|+| F\left(z_{i}, y^{\prime}\right)-F\left(z_{i}, y_{i}\right)\left|\leq K^{\prime} \delta+\right| F\left(z_{i}, y_{i}^{\prime}\right)-F\left(z_{i}, y_{i}\right) \mid$ (where $K^{\prime}$ is $\sqrt{p}$ times a Lipschitz constant of $\left.F\right|_{V}$ which we may suppose to exist) $\leq K^{\prime} \delta+\left|G\left(z_{i}, y_{i}^{\prime}\right)-G\left(z_{i}, y_{i}\right)\right|$.

Observe now that $\left(z_{i}, y_{i}\right)=\left(z_{i}, \widetilde{\psi}_{i}\left(p_{1}\right)\right)$ and $\left(z_{i}^{\prime}, y_{i}^{\prime}\right)=\left(z_{i}, \widetilde{\psi}_{i}\left(p_{2}\right)\right)$, for some $p_{1}, p_{2}$ in $\left\{z_{i}\right\} \times B_{i}$ with $\left|p_{1}-p_{2}\right| \leq\left|y_{i}-y_{i}^{\prime}\right| \leq 2 \varepsilon \sqrt{n}$. Let $\gamma:[0,1] \rightarrow$ $V_{i} \times B_{i}$ be a straight path joining $p_{1}$ and $p_{2}$. Then $G\left(z_{i}, y_{i}^{\prime}\right)-G\left(z_{i}, y_{i}\right)=$ $\int_{0}^{1} \frac{\partial G}{\partial y}(\widetilde{\gamma}(t)) \cdot \widetilde{\gamma}^{\prime}(t) d t$, where $\widetilde{\gamma}:=\psi_{i} \circ \gamma$. We have $\frac{\partial G}{\partial y}(\widetilde{\gamma}(0))=0$, so

$$
\begin{aligned}
\left\|\frac{\partial G}{\partial y}(\widetilde{\gamma}(t))\right\| & =\left\|\frac{\partial G}{\partial y}(\widetilde{\gamma}(t))-\frac{\partial G}{\partial y}(\widetilde{\gamma}(0))\right\| \\
& \leq M\left|p_{1}-p_{2}\right|^{k+\alpha-1} \\
& \leq M(2 \varepsilon \sqrt{n})^{k+\alpha-1} \Rightarrow\left\|\frac{\partial G}{\partial y}(\widetilde{\gamma}(t))\right\|\left|\gamma^{\prime}(t)\right| \\
& \leq K^{\prime \prime} \varepsilon^{k+\alpha},
\end{aligned}
$$

for some constant $K^{\prime \prime}$. Indeed, $\left|\widetilde{\gamma}^{\prime}(t)\right|$ is limited by a constant multiple of $\left|p_{1}-p_{2}\right| \leq 2 \sqrt{n} \varepsilon$. So

$$
\left|G\left(z_{1}, y_{i}^{\prime}\right)-G\left(z_{i}, y_{i}\right)\right| \leq \int_{0}^{1}\left|\frac{\partial G}{\partial y}(\widetilde{\gamma}(t)) \circ \widetilde{\gamma}(t)\right| d t \leq K^{\prime \prime} \varepsilon^{k+\alpha}
$$


and

$$
\left|F\left(z_{i}^{\prime}, y^{\prime}\right)-F\left(z_{i}, y_{i}\right)\right| \leq K^{\prime} \delta+K^{\prime \prime} \varepsilon^{k+\alpha} \leq K_{0}^{\prime} \cdot \varepsilon^{k+\alpha}
$$

where $K_{0}^{\prime}=K^{\prime}+K^{\prime \prime}$.

Therefore, $F\left(C_{\delta}\left(\widetilde{z}_{i}\right) \times C_{\varepsilon}(y)\right)$ is contained in some ball $B_{\delta_{i}}\left(q_{i}\right)$, with $\delta_{i} \leq K_{0}^{\prime} \cdot \varepsilon^{k+\alpha}$, so

$$
\begin{aligned}
\sum_{i} \delta_{i}^{d} & \leq\left(\left[\varepsilon^{1-(k+\alpha)}\right]+1\right)^{p}\left(K_{0}^{\prime} \varepsilon^{k+\alpha}\right)^{d} \\
& =\left(K_{0}^{\prime}\right)^{d}\left(\left[\varepsilon^{1-(k+\alpha)}\right]+1\right)^{p}\left(\varepsilon^{k+\alpha}\right)^{p+\frac{n-p}{k+\alpha}} \\
& \leq \widetilde{K}_{0} \varepsilon^{n}
\end{aligned}
$$

for some constant $\widetilde{K}_{0}$. So, $F\left(C_{\varepsilon}(x)\right)$ can be covered by balls $B_{\delta_{i}}\left(q_{i}\right)$ with $\sum_{i} \delta_{i}^{d} \leq K_{0} \cdot \lambda\left(C_{\varepsilon}(x)\right)$, and by the Lemma 3.2, Remarks 3.2 and 3.4, we can conclude that $m_{d}\left(F\left(A_{i} \cap X\right)\right) \leq K_{0} \lambda(X)$ where $K_{0}=2^{n} \widetilde{K}_{0}$, and so we can cover $F\left(A_{i} \cap X\right)$ by balls $B_{\delta_{i}}\left(p_{i}\right)$ so that $\sum_{i=1}^{\infty} \delta_{i}^{d} \leq K_{0}(\lambda(X)+\nu)$, as we stated.

We shall prove now that there is an $A_{i}^{\prime} \subset A_{i} \subset V$ with $\lambda\left(A_{i} \backslash A_{i}^{\prime}\right)=$ 0 such that $F\left(C_{\varepsilon}(x) \cap A_{i}\right)$ can be covered by balls $B_{\delta_{i}^{(\varepsilon)}}\left(W_{i}\right), i \in \mathbb{N}$ with $\lim _{\varepsilon \rightarrow 0} \frac{\sum_{i}\left(\delta_{i}^{(\varepsilon)}\right)^{d}}{\lambda\left(C_{\varepsilon}(x)\right)}=0, \forall x \in A_{i}^{\prime}$. This will imply our theorem, by the Lemma 3.2, Remarks 3.2 and 3.3, since we have proved above that $m_{d}\left(F\left(C_{\varepsilon}(x) \cap A_{i}\right) \leq K_{0} \lambda\left(C_{\varepsilon}(x)\right), \forall \varepsilon<\frac{1}{2 \sqrt{n} M}\right.$. For this, since $A_{i} \subset$ $\psi_{i}\left(V_{i} \times B_{i}\right), B_{i} \subset \mathbb{R}^{r_{i}}, r_{i} \leq n-p$, we may suppose $r_{i}=n-p$ and $\psi_{i}=$ identity, because $r_{i}<n-p \Rightarrow \lambda\left(A_{i}\right)=0$ and we can take $A_{i}^{\prime}=A_{i}$. Let us take $A_{i}^{\prime}$ equal to the set of the density points of $A_{i}$. Given a point $x \in A_{i}^{\prime}$, and an $\eta^{\prime}>0$, we want to find an $\varepsilon_{0}>0$ such that $\varepsilon<\varepsilon_{0} \Rightarrow F\left(C_{\varepsilon}(x) \cap A_{i}\right)$ can be covered by balls $B_{\delta_{i}^{(\varepsilon)}}\left(W_{i}\right), i \in \mathbb{N}$ such that $\sum_{i}\left(\delta_{i}^{(\varepsilon)}\right)^{d} \leq \eta^{\prime} \lambda\left(C_{\varepsilon}(x)\right)$. Let $\eta, \tilde{\eta}>0, \tilde{\varepsilon}<\frac{1}{2 \sqrt{n} M}$ such that $\frac{\lambda\left(C_{\varepsilon}(x) \cap A_{i}\right)}{\lambda\left(C_{\varepsilon}(x)\right)}>1-\tilde{\eta}^{2}, \forall \varepsilon \leq \tilde{\varepsilon}$. Divide the cube $C_{\varepsilon}(x)=C_{\varepsilon}(\widetilde{z}) \times C_{\varepsilon}(\widetilde{y})$, $\varepsilon<\tilde{\varepsilon}$ into $N=\left(\left[\varepsilon^{1-(K+\alpha)} \eta^{-1}\right]+1\right)^{p}$ boxes $C_{\delta}\left(\widetilde{z}_{i}\right) \times C_{\varepsilon}(\widetilde{y}), \delta<\eta \varepsilon^{k+\alpha}$, $1 \leq i \leq N$. Then for at least $(1-\tilde{\eta}) N$ values of $i$, there is a $z_{i} \in C_{\delta}\left(\widetilde{z}_{i}\right)$ such that $\lambda\left(\left\{y \in C_{\varepsilon}(\widetilde{y}) \mid\left(z_{i}, y\right) \in A_{i}\right\} / \lambda\left(C_{\varepsilon}(\widetilde{y})\right)>1-\tilde{\eta}\right.$ (here $\lambda$ is the Lebesgue measure in $\left.\mathbb{R}^{n-p}\right)$, because $\lambda\left(C_{\varepsilon}(x) \cap A_{i}\right)>\left(1-\tilde{\eta}^{2}\right) \lambda\left(C_{\varepsilon}(x)\right)$. 
For such an $i$, take an $y_{i}$ such that $\left(z_{i}, y_{i}\right) \in A_{i}$. Then, applying Theorem 2.1, Corollaries 2.2 and 2.3, given $\eta$ we can choose $\tilde{\eta}$ so that $\left|F\left(z_{i}, y\right)-F\left(z_{i}, y_{i}\right)\right|<\eta \cdot \varepsilon^{k+\alpha} \Rightarrow\left|F(z, y)-F\left(z_{i}, y_{i}\right)\right| \leq 2 K^{\prime} \sqrt{n} . \eta \varepsilon^{K+\alpha}+$ $\eta \varepsilon^{K+\alpha} \leq K^{\prime \prime} \eta \varepsilon^{k+\alpha}$, for some constant $K^{\prime \prime}$ and for any $z \in C_{\varepsilon}\left(\widetilde{z}_{i}\right)$, where $K^{\prime}$ is a Lipschitz constant for $F \Rightarrow F\left(C_{\delta}\left(\widetilde{z}_{i}\right) \times C_{\varepsilon}(\widetilde{y})\right)$ is contained in a ball $B_{\delta_{i}}\left(Q_{i}\right)$, with $\sum_{i} \delta_{i}^{d}$ over these values of $i$ less than

$$
\begin{aligned}
& \left(\left[\varepsilon^{1-(K+\alpha)} \eta^{-1}\right]+1\right)^{p}\left(K^{\prime \prime} \eta \varepsilon^{k+\alpha}\right)^{d} \\
& \quad=\left(K^{\prime \prime}\right)^{d}\left(\left[\varepsilon^{1-(k+\alpha)} \cdot \eta^{-1}\right]+1\right)^{p}\left(\eta \varepsilon^{k+\alpha}\right)^{p+\frac{n-p}{k+\alpha}} \leq \widetilde{K}_{0} \eta^{\frac{n-p}{k+\alpha}} \cdot \varepsilon^{n}
\end{aligned}
$$

for some constant $\widetilde{K}_{0}$. The union of the remaining (at most $\tilde{\eta} N$ ) boxes has volume at most $\tilde{\eta} \varepsilon^{n} \Rightarrow$ the union of the image of the intersection of $A_{i}$ with the union of these boxes by $F$ is contained in a union of balls $B_{\delta_{i}}\left(Q_{i}\right)$ with $\sum_{i} \delta_{i}^{d} \leq 2 K_{0} \tilde{\eta} \varepsilon^{n}$, by the statement proved before, and so $F\left(C_{\varepsilon}(x)\right)$ can be covered by balls $B_{\delta_{i}}\left(\widetilde{Q}_{i}\right)$ with $\Sigma \delta_{i}^{d} \leq\left(\widetilde{K}_{0} \eta^{\frac{n-p}{k+\alpha}}+\right.$ $\left.2 K_{0} \tilde{\eta}\right) \varepsilon^{n}$. Choosing $\eta, \tilde{\eta}$ so small that $\widetilde{K}_{0} \eta^{\frac{n-p}{k+\alpha}}+2 K_{0} \tilde{\eta} \leq \eta^{\prime}$, we obtain the desired result with $\varepsilon_{0}=\tilde{\varepsilon}$.

Remark 3.5. In the cases of functions of class $C^{k}\left(C^{k+\alpha}\right.$ with $\left.\alpha=0\right)$ we have the same result. If $k \geq 2$, it follows from the case of class $C^{k-1+(1)}$ of the theorem. If $k=1, p+\frac{n-p}{k+\alpha}=n$, and the proof of the Theorem 3.3 shows that if $F: \mathbb{R}^{n} \rightarrow \mathbb{R}^{m}$ is a function and $C(F)=\{x \in U$ $D F(x)$ exists and $\operatorname{rank} D F(x)<n\}$ then $m_{n}(F(C(F)))=0$, where $m_{n}$ is the Hausdorff measure of dimension $n$.

\section{Examples}

In this section we give some examples which show that the previous results are quite sharp. In all these examples we shall use a certain kind of functions of the real line that we shall describe below.

Definition 4.1. Let $\left(\lambda_{n}\right)_{n \in \mathbb{N}}$ be a sequence of real numbers with $0<$ $\lambda_{i}<\frac{1}{2}, \forall i \in \mathbb{N}$. The central Cantor set $K_{\underline{\lambda}}$ is the Cantor set constructed as follows: We remove from the interval $[0,1]$ the central open interval $U_{1,1}$ of proportion $1-2 \lambda_{1}$, then we remove from the two remaining intervals the central open intervals $U_{2,1}$ and $U_{2,2}$ of proportion $1-2 \lambda_{2}$, and so on. After the $r$-th step of the construction there will remain $2^{r}$ intervals of length $\lambda_{1} \lambda_{2} \ldots \lambda_{r}$. The intersection of all these sets is the central Cantor set $K_{\underline{\lambda}}$. The open intervals removed in the $r$-th step of the construction have length $\lambda_{1} \lambda_{2} \ldots \lambda_{r-1}\left(1-2 \lambda_{r}\right)$. 
Let $\psi: \mathbb{R} \stackrel{C^{\infty}}{\longrightarrow} \mathbb{R}$ be a fixed function such that $\psi(\mathbb{R}) \subset[0,1], \psi(x)=0$, $\forall x \leq 0, \psi(x)=1, \forall x \geq 1$. Given two central Cantor sets $K_{\lambda}$ and $K_{\mu}$, we construct the function $f_{\underline{\lambda}, \mu}: \mathbb{R} \rightarrow \mathbb{R}$ as follows: $f_{\underline{\lambda}, \mu}(x)=0$, $\forall x \leq 0, f_{\underline{\lambda}, \mu}(x)=1, \forall x \geq 1$, and if $U_{i, j}=(a, b)$ and $v_{i, j}=(c, d)$ are corresponding removed intervals in the constructions of $K_{\lambda}$ and $K_{\mu}$, respectively, we define $f_{\underline{\lambda}, \underline{\mu}}(x)=c+(d-c) \psi\left(\frac{x-a}{b-a}\right), \forall x \in(a, b)$. We extend $f_{\underline{\lambda}, \mu}$ to $K_{\underline{\lambda}}$ by continuity, obtaining $f_{\underline{\lambda}, \mu}\left(K_{\underline{\lambda}}\right)=K_{\mu}$. It is easy to check that if $g_{r}:=\lambda_{1} \lambda_{2} \ldots \lambda_{r-1}\left(1-2 \lambda_{r}\right)$ and $\tilde{g}_{r}:=\mu_{1} \mu_{2} \ldots \mu_{r-1}\left(1-2 \mu_{r}\right)$ satisfy $\lim _{r \rightarrow \infty} \frac{\tilde{g}_{r}}{g_{r}^{k}}=0$ then $f_{\underline{\lambda}, \underline{\underline{\mu}}}$ is $C^{k}$ (if $k \geq 1$ is an integer). Moreover, if $q>1$, and $\sup \rightarrow \frac{\tilde{g}_{r}}{g_{r}^{q}}<\infty$ then $f_{\underline{\lambda}, \underline{\mu}}$ is $C^{q-1,1}$ if $q$ is integer and is $C^{q}$ (i.e., it is $C^{[q]+\{q\}}$, where $\left.\{q\}=q-[q] \in(0,1)\right)$ otherwise. See [BMPV] for more details.

Example 4.1. Let $\lambda_{n}=\frac{1}{2}-\frac{1}{2 n}, \mu_{n} \equiv a$. Then $\lim _{n \rightarrow \infty} \frac{\tilde{g}_{n}}{g_{n}^{q}}=0, \forall q<$ $\frac{-\log a}{\log 2}$, and so $f_{\underline{\lambda}, \underline{\mu}}$ is $C^{q}, \forall q<\frac{-\log a}{\log 2}$. On the other hand, $m_{d}\left(K_{\underline{\mu}}\right)=1$ where $d=\frac{-\log 2}{\log a}$ (see [PT]). Moreover, since $a \in\left(0, \frac{1}{2}\right), \lim _{n \rightarrow \infty} \frac{\tilde{g}_{n}^{-}}{g_{n}}=0$, and so $f_{\underline{\lambda}, \underline{\mu}}^{\prime}(x)=0, \forall x \in K_{\underline{\lambda}}$. If $F: \mathbb{R}^{n+p} \rightarrow \mathbb{R}^{n+p}$ is given by

$$
\begin{array}{r}
F\left(x_{1}, x_{2}, \ldots, x_{n}, x_{n+1}, \ldots, x_{n+p}\right) \\
=\left(f_{\underline{\lambda}, \underline{\mu}}\left(x_{1}\right), f_{\underline{\lambda}, \underline{\mu}}\left(x_{2}\right) \ldots, f_{\underline{\lambda}, \underline{\mu}}\left(x_{n}\right), x_{n+1} \ldots, x_{n+p}\right),
\end{array}
$$

then

$F\left(C_{p}(F)\right)=F\left(K_{\underline{\lambda}} \times K_{\underline{\lambda}} \times \cdots \times K_{\underline{\lambda}} \times \mathbb{R}^{p}\right)=K_{\mu} \times K_{\mu} \times \cdots \times K_{\underline{\mu}} \times \mathbb{R}^{p}$

that is a set with positive $(n d+p)$-measure. This shows that given $q>1$, $p>0$ and $n>p$ there is a map $F: \mathbb{R}^{n} \rightarrow \mathbb{R}^{n}$ such that $m_{d}\left(F\left(C_{p}(F)\right)\right)>$ 0 , where $d=p+\frac{n-p}{q}$, and $F$ is of class $C^{q^{\prime}}$ for each $q^{\prime}<q$.

Remark 4.1. If $a=\frac{1}{2^{n}}, F\left(x_{1}, x_{2}, \ldots, x_{n}\right)=f_{\underline{\lambda}, \underline{\mu}}\left(x_{1}\right)+2 f_{\underline{\lambda}, \underline{\mu}}\left(x_{2}\right)+\cdots+$ $2^{n-1} f_{\underline{\lambda}, \underline{\mu}}\left(x_{n}\right)$ gives an example of a function $F: \mathbb{R}^{n} \rightarrow \mathbb{R}$ which is of class $\bar{C}^{\bar{q}}, \forall q<n(q \in \mathbb{R})$ such that $F\left(\mathbb{R}^{n}\right)$ contains an open set, since $K_{\mu}+2 K_{\mu}+\cdots+2^{n-1} K_{\mu}=\left[0,2^{n}-1\right]$, which can be proved easily using representation in basis $2^{\bar{n}}$.

Example 4.2. Let $\lambda_{n}=\frac{1}{2}-\frac{1}{3 n^{2}}, \mu_{n}=a-\frac{a}{3 n}, a \in(0,1 / 2]$. Then $\lim _{n \rightarrow \infty} \frac{\tilde{g}_{n}}{g_{n}^{q}}=0$, where $q=\frac{-\log a}{\log 2}$, and so $f_{\underline{\lambda}, \underline{\mu}}$ is $C^{q}$. On the other hand we have $H D\left(K_{\underline{\mu}}\right) \geq \frac{-\log 2}{\log a}$. Indeed, if $b<a$ and $\theta_{n} \equiv b, f_{\underline{\mu}, \underline{\theta}}$ is clearly $C^{1}$, and $f_{\underline{\mu}, \underline{\theta}}\left(K_{\underline{\mu}}\right)=K_{\underline{\theta}} \Rightarrow H D\left(K_{\underline{\mu}}\right) \geq H D\left(K_{\underline{\theta}}\right)=\frac{-\log 2}{\log b}, \forall b<a$. If 
$F: \mathbb{R}^{n+p} \rightarrow \mathbb{R}^{n+p}$ is given by

$$
\begin{aligned}
F\left(x_{1}, x_{2}, \ldots, x_{n}, x_{n+1}, \ldots, x_{n+p}\right) & \\
& =\left(f_{\underline{\lambda}, \underline{\mu}}\left(x_{1}\right), \ldots, f_{\underline{\lambda}, \underline{\mu}}\left(x_{n}\right), x_{n+1}, \ldots, x_{n+p}\right)
\end{aligned}
$$

then

$$
F\left(C_{p}(F)\right)=K_{\underline{\mu}} \times \cdots \times K_{\underline{\mu}} \times \mathbb{R}^{p},
$$

that is a set with Hausdorff dimension $n d+p$, where $d=\frac{-\log 2}{\log a}$. This shows that given $q \geq 1, p>0$ and $n>p$ there is a map $F: \mathbb{R}^{n} \rightarrow \mathbb{R}^{n}$ such that $H D\left(F\left(C_{p}(F)\right)\right)=p+\frac{n-p}{q}$, and $F$ is of class $C^{q}$.

\section{References}

[BMPV] R. Bamón, C. G. Moreira, S. Plaza and J. Vera, Differentiable structures of central Cantor sets, Ergodic Theory Dynam. Systems 17(5) (1997), 1027-1042.

[B1] S. M. BAtes, On the image size of singular maps. II, Duke Math. J. 68(3) (1992), 463-476.

[B2] S. M. BATES, Toward a precise smoothness hypothesis in Sard's theorem, Proc. Amer. Math. Soc. 117(1) (1993), 279-283.

[Ch1] P. T. Church, Differentiable open maps on manifolds, Trans. Amer. Math. Soc. 109 (1963), 87-100.

[Ch2] P. T. Church, On points of Jacobian rank $k$. II, Proc. Amer. Math. Soc. 16 (1965), 1035-1038.

[Co] A. Constantin, Critical values of differentiable maps, Exposition. Math. 14(1) (1996), 93-95.

[F] H. FEDERER, "Geometric measure theory", Die Grundlehren der mathematischen Wissenschaften 153, Springer-Verlag New York Inc., New York, 1969.

[M] A. P. Morse, The behavior of a function on its critical set, Ann. of Math. 40 (1939), 62-70.

[PT] J. PAlis And F. TAKEns, "Hyperbolicity and sensitive chaotic dynamics at homoclinic bifurcations", Cambridge Studies in Advanced Mathematics 35, Cambridge University Press, Cambridge, 1993.

[S1] A. SARD, The measure of the critical values of differentiable maps, Bull. Amer. Math. Soc. 48 (1942), 883-890.

[S2] A. SARD, Hausdorff measure of critical images on Banach manifolds, Amer. J. Math. 87 (1965), 158-174. 
[Y] Y. Yomdin, The geometry of critical and near-critical values of differentiable mappings, Math. Ann. 264(4) (1983), 495-515.

IMPA

Estrada Dona Castorina, 110

22460-320, Río de Janeiro, RJ

Brasil

E-mail address: gugu@impa.br

Primera versió rebuda el 24 d'abril de 2000, darrera versió rebuda el 17 d'octubre de 2000. 